\author{
P. Flachs $\cdot$ V. Mohamed-Ali $\cdot$ O. Horakova $\cdot$ \\ M. Rossmeisl • M. J. Hosseinzadeh-Attar • \\ M. Hensler · J. Ruzickova $\cdot$ J. Kopecky
}

\title{
Polyunsaturated fatty acids of marine origin induce adiponectin in mice fed a high-fat diet
}

Received: 30 June 2005 / Accepted: 30 August 2005 / Published online: 6 January 2006

C) Springer-Verlag 2006

\begin{abstract}
Aims/hypothesis: Diets rich in $n-3$ polyunsaturated fatty acids, namely eicosapentaenoic acid (EPA) and docosahexaenoic acid (DHA), protect against insulin resistance and obesity in rodents and increase insulin sensitivity in healthy humans. We tested whether the anti-diabetic effects of EPA and DHA involve enhanced production of the endogenous insulin sensitiser, adiponectin. Methods: We studied the effects, in an obesity-promoting high-fat diet, of partial replacement of vegetable oils by EPA/DHA concentrate $(6 \% \mathrm{EPA}, 51 \% \mathrm{DHA})$ over a 5 -week period in adult male C57BL/6J mice that either had free access to food or had their food intake restricted by $30 \%$. At the end of the treatment, systemic markers of lipid and glucose metabolism and full-length adiponectin and leptin were measured. Adiponectin (Adipoq) and leptin (Lep) gene expression in dorsolumbar and epididymal white adipose tissue (WAT) and isolated adipocytes was quantified and adipokine production from WAT explants evaluated. Results: In mice with free access to food, plasma triacylglycerols, NEFA, and insulin levels were lower in the presence of EPA/DHA, while glucose and leptin levels were not significantly altered. Food restriction decreased plasma triacylglycerols, glucose, insulin and leptin, but not adiponectin. EPA/DHA increased plasma adiponectin levels, independent of food intake, reflecting the stimulation of Adipoq expression in adipocytes and the release of adiponectin from WAT, particularly from epididymal fat. Expression of Lep and the
\end{abstract}

P. Flachs $\cdot$ O. Horakova $\cdot$ M. Rossmeisl

M. Hensler · J. Ruzickova · J. Kopecky $(\bowtie)$

Department of Adipose Tissue Biology,

Institute of Physiology, Academy of Sciences,

Videnska 1083

142 20, Prague, Czech Republic

e-mail: kopecky@biomed.cas.cz

Tel.: +420-241-062554

Fax: +420-241-062599

V. Mohamed-Ali · M. J. Hosseinzadeh-Attar Adipokines and Metabolism Research Group, Department of Medicine, University College London, London, UK release of leptin from WAT, while being extremely sensitive to caloric restriction, was unaltered by EPA/DHA. Conclusions/interpretation: Intake of diets rich in EPA and DHA leads to elevated systemic concentrations of adiponectin, largely independent of food intake or adiposity and explain, to some extent, their anti-diabetic effects.

Keywords Adipokines - Adiponectin - Adipose tissue . Fish oil $\cdot$ High-fat diet $\cdot n-3$ PUFA

Abbreviations Adipoq: Gene encoding adiponectin . AMPK: AMP-activated protein kinase - cHF diet: Composite high-fat diet based on chow $\cdot$ cHF-F1 diet: Composite high-fat diet with partial replacement of lipids by fish oil concentrate - DHA: Docosahexaenoic acid . EPA: Eicosapentaenoic acid - EPA/DHA: Concentrate of EPA and DHA from sea fish (6\% EPA and 51\% DHA) . Lep: Gene encoding leptin $\cdot$ Ppib: Gene encoding peptidylpropyl isomerase $\beta$, also called Cyclophilin B . PUFA: Polyunsaturated fatty acids - qRT-PCR:

Quantitative real-time RT-PCR - Slc2a4: Gene encoding solute carrier family 2 member 4, also called Glut4 - WAT: White adipose tissue.

\section{Introduction}

Intake of polyunsaturated fatty acids (PUFA) of marine origin, namely eicosapentaenoic acid (EPA; 20:5n-3) and docosahexaenoic acid (DHA, 22:6 n-3), has numerous beneficial effects on health. EPA and DHA act as hypolipidaemics, exert prophylactic effects on cardiovascular disease, protect against insulin resistance and obesity in rodents fed high-fat diets, and reduce insulin response to glucose in healthy humans [1-3 and references therein]. We, and others, have shown [1] that in a semi-synthetic high-fat diet rich in $\alpha$-linolenic acid $(18: 3 n-3)$ partial replacement of lipids with concentrate of EPA and DHA from sea fish (EPA/DHA) resulted in suppression of insulinaemia. It also protected against down-regulation of solute carrier family 2 member 4 (Slc2a4, also called Glut 4 ) 
in white adipose tissue (WAT) by the high-fat diet. The effect on Slc2a4 was part of a complex modulation of WAT gene expression and metabolism, resulting in stimulation of lipid oxidation and inhibition of lipogenesis, especially in the epididymal fat, which led to reduced accumulation of this tissue while food consumption remained unaffected $[1,4]$.

The aim of this work was to test the hypothesis that the anti-diabetic effects of EPA and DHA involve induction of adiponectin, an adipocyte-derived hormone, which stimulates glucose utilisation and fatty acid oxidation in muscles and decreases hepatic gluconeogenesis as a result of the activation of AMP-activated protein kinase (AMPK) [5]. We show that partial replacement of dietary lipids with EPA/DHA in a composite high-fat (cHF) diet in rodents stimulates expression of the gene encoding adiponectin (Adipoq) and increases adiponectin production in epididymal WAT as well as systemic levels of adiponectin by mechanism(s) relatively independent of food intake or adiposity.

\section{Materials and methods}

Animals and diets Experiments were performed on adult male $\mathrm{C} 57 \mathrm{BL} / 6 \mathrm{~J}$ mice as described previously $[1,4]$. Mice were imported from the Jackson Laboratory (Bar Harbor, ME, USA) and bread at the Institute of Physiology for up to 16 generations. The animals studied were habituated for 2 weeks to the cHF diet, which was derived from standard chow and contained 35\% (wt/wt) lipids of very low $n-3$ PUFA content, and then assigned for 5 weeks to the cHF diet, or a diet of the same composition, i.e. a composite high-fat diet, but with partial replacement of lipids by fish oil concentrate (cHF-F1 diet) such that $15 \%(\mathrm{wt} / \mathrm{wt})$ of lipids was replaced by the EPA/DHA concentrate containing $6 \% \mathrm{EPA}, 51 \% \mathrm{DHA}$, and $4 \mathrm{mg} / \mathrm{g} \alpha$-tocopherol as antioxidant (EPAX 1050TG; Pronova Biocare, Lysaker, Norway). Detailed analysis of fatty acid composition of the diets was performed previously [1]. When indicated, the ration was reduced by $30 \%$ (caloric restriction) compared with mice on the same type of diet but with free access to chow. Mice were killed by cervical dislocation, and subcutaneous dorsolumbar and epididymal WAT depots were dissected. Tissues were used immediately for explant studies or isolation of adipocytes [4], or stored in liquid nitrogen for RNA analysis (see below). EDTA plasma was obtained from truncal blood and stored at $-70^{\circ} \mathrm{C}$. The experiments were conducted according to the Institute's guidelines for the use and care of laboratory animals.

WAT explants Tissue $(100 \mathrm{mg})$ was incubated in $1.0 \mathrm{ml}$ of serum-free medium (Cellgro; Mediatech, Herndon, VA, USA) for $24 \mathrm{~h}$ at $37^{\circ} \mathrm{C} / 5 \% \mathrm{CO}_{2}$. At the end of the incubation the culture supernatant was stored at $-70^{\circ} \mathrm{C}$.

Quantification of metabolites and hormones in plasma and supernatant from white adipose tissue explants Triacylglycerol, NEFA, glucose and insulin were estimated as described before [1]. Leptin and full-length adiponectin were measured by 2-site ELISA ( $\mathrm{R} \& \mathrm{D}$ Systems, Minneapolis, MN, USA) with inter- and intra-assay CVs of 5.8 and 8.9 for leptin and 6.3 and $9.4 \%$ for adiponectin.

Quantitative real-time $R T-P C R$ Total RNA was isolated as before [4] and quantitative real-time RT-PCR (qRT-PCR) was performed using a qPCR kit (DyNAmoCapillary SYBRGreen; Finnzymes, Espoo, Finland) and LightCycler (Hoffman-La Roche, Basel, Switzerland). Levels of transcripts were standardised using the gene encoding peptidylpropyl isomerase B (Ppib, also called cyclophilin B) and expressed in arbitrary units [4]. Lasergene software (DNASTAR, Madison, WI, USA) was used to design oligonucleotide primers (forward/reverse) for the following genes: Adipoq - TCCGGGACTCTACTACTTCTCTTAC CAC / GTCCCCATCCCCATACACCTG; Ppib-ACTAC GGGCCTGGCTGGGTGAG / TGCCGGAGTCGACAA

Table 1 Effects of EPA/DHA and caloric restriction on body weight, markers of lipid and glucose metabolism, and adipokines in plasma

\begin{tabular}{|c|c|c|c|c|c|}
\hline & \multicolumn{4}{|c|}{ Diet } & \multirow[b]{3}{*}{$n$} \\
\hline & \multicolumn{2}{|c|}{ Free access to food } & \multicolumn{2}{|c|}{ Caloric restriction } & \\
\hline & $\mathrm{cHF}$ & $\mathrm{cHF}-\mathrm{F} 1$ & $\mathrm{cHF}$ & $\mathrm{cHF}-\mathrm{F} 1$ & \\
\hline Body weight $(\mathrm{g})^{\mathrm{a}}$ & $37.5 \pm 1.2$ & $34.8 \pm 1.2^{\mathrm{b}}$ & $25.1 \pm 0.4^{\mathrm{c}}$ & $25.2 \pm 0.6^{\mathrm{c}}$ & 10 \\
\hline Plasma levels of: & - & - & - & - & - \\
\hline NEFA (mmol/l) & $0.47 \pm 0.02$ & $0.33 \pm 0.02^{\mathrm{b}}$ & $0.44 \pm 0.05$ & $0.38 \pm 0.05$ & 10 \\
\hline Triacylglycerols (mmol/1) & $1.46 \pm 0.11$ & $0.79 \pm 0.07^{\mathrm{b}}$ & $0.51 \pm 0.06^{\mathrm{c}}$ & $0.52 \pm 0.05^{\mathrm{c}}$ & 10 \\
\hline Glucose $(\mathrm{mmol} / \mathrm{l})$ & $10.10 \pm 0.22$ & $10.04 \pm 0.38$ & $7.55 \pm 0.44^{\mathrm{c}}$ & $7.27 \pm 0.33^{\mathrm{c}}$ & 10 \\
\hline Insulin $(\mathrm{ng} / \mathrm{ml})$ & $2.77 \pm 0.52$ & $0.17 \pm 0.04^{\mathrm{b}}$ & $1.68 \pm 0.22^{\mathrm{c}}$ & $0.16 \pm 0.06^{\mathrm{b}}$ & 7 \\
\hline Adiponectin $(\mu \mathrm{g} / \mathrm{ml})$ & $9.0 \pm 0.3$ & $12.1 \pm 0.5^{\mathrm{b}}$ & $9.2 \pm 1.1$ & $11.2 \pm 0.5^{\mathrm{b}}$ & 10 \\
\hline Leptin $(\mathrm{ng} / \mathrm{ml})$ & $42.5 \pm 7.9$ & $40.1 \pm 0.9$ & $2.4 \pm 0.4^{\mathrm{c}}$ & $2.5 \pm 0.2^{\mathrm{c}}$ & 10 \\
\hline
\end{tabular}

Mice had either free access to $\mathrm{cHF}$ or $\mathrm{cHF}-\mathrm{F} 1$ diets or were subject to caloric restriction. Data are means \pm SEM. ${ }^{\mathrm{a}}$ Animals were used in our previous study [1]. ${ }^{\mathrm{b}}$ Significant effect of diet. ${ }^{\mathrm{c}}$ Significant effect of caloric restriction 
Table 2 Gene expression and adipokine production in WAT depots of mice with free access to cHF or cHF-F1 diets

\begin{tabular}{|c|c|c|c|c|c|}
\hline & \multicolumn{2}{|c|}{ Dorsolumbar fat } & \multicolumn{2}{|c|}{ Epididymal fat } & \multirow[b]{2}{*}{$n$} \\
\hline & $\mathrm{cHF}$ & cHF-F1 & $\mathrm{cHF}$ & $\mathrm{cHF}-\mathrm{F} 1$ & \\
\hline Gene expression & - & - & - & - & - \\
\hline Tissue & - & - & - & - & - \\
\hline Adipoq (AU) & $0.77 \pm 0.08$ & $0.94 \pm 0.07$ & $0.82 \pm 0.03$ & $0.88 \pm 0.06$ & 10 \\
\hline Lep (AU) & $0.51 \pm 0.05$ & $0.48 \pm 0.03$ & $0.61 \pm 0.04$ & $0.47 \pm 0.07$ & 10 \\
\hline Adipocytes & - & - & - & - & - \\
\hline Adipoq (AU) & $3.21 \pm 0.25$ & $6.13 \pm 0.87^{\mathrm{b}}$ & $2.83 \pm 0.23$ & $7.18 \pm 0.82^{\mathrm{b}}$ & $7-8$ \\
\hline Lep (AU) & $2.92 \pm 0.83$ & $3.57 \pm 0.91$ & $1.70 \pm 0.40$ & $3.11 \pm 1.06$ & $7-8$ \\
\hline Adipokine production ${ }^{\mathrm{a}}$ & - & - & - & - & - \\
\hline Adiponectin $(\mu \mathrm{g} / \mathrm{ml})$ & $0.31 \pm 0.04$ & $0.32 \pm 0.03$ & $0.32 \pm 0.03$ & $0.47 \pm 0.03^{\mathrm{b}}$ & 9 \\
\hline Leptin (ng/ml) & $36.1 \pm 3.1$ & $34.7 \pm 3.6$ & $33.6 \pm 6.4$ & $20.9 \pm 3.3$ & 9 \\
\hline
\end{tabular}

Transcript levels were evaluated by qRT-PCR using RNA isolated from whole tissues or collagenase-liberated adipocytes. Tissue explants were used to measure adipokine production. Data are means \pm SEM. ${ }^{a}$ Levels in medium after incubation of WAT explants (see Materials and methods). ${ }^{\text {b }}$ Significant effect of diet. AU, arbitrary units

TGATGA; and leptin (Lep) - CCGCCAAGCAGAGGGT CAC / GCATTCAGGGCTAACATCCAACT.

Statistics The data were evaluated by ANOVA as described before [4]. The level of significance of all tests was set at $p=0.05$.

\section{Results}

Mice that had free access to cHF or cHF-F1 diets or a calorie-restricted diet for 5 weeks were compared (Table 1). In accordance with our previous results in mice with free access to standard chow [1, 4], the cHF diet induced obesity, while the cHF-F1 diet resulted in $2.7 \mathrm{~g}$ lower mean body weight and about $30 \%$ reduction of epididymal fat mass, with no change in dorsolumbar fat. Under these conditions, food consumption was not affected [4]. Caloric restriction resulted in net loss of body weight and adipose tissue mass [1, 4] (Table 1). Plasma levels (Table 1) of NEFA were lower in cHF-F1 than cHF mice with free access to food, but they were not affected by the diet in animals on the calorie-restricted diet, and no independent effect of caloric restriction on NEFA levels was seen. In mice with free access to food, levels of triacylglycerols were lower in cHF-F1 than in cHF mice, but the effect of the cHF-F1 diet was masked by caloric restriction, which decreased triacylglycerol levels (Table 1). Glucose and leptin levels were not affected by the cHF-F1 diet, but they were reduced by caloric restriction. Insulin levels were substantially lower in cHF-F1 than cHF mice, independently of food intake. Also caloric restriction decreased insulin levels, but to a much lower degree than the decrease induced by cHF-F1 diet. Levels of adiponectin were increased by cHF-F1 diet to a similar extent in mice with free access to food as in the mice on a calorie-restricted diet (34 and 22\%, respectively), with this effect being independent of caloric restriction.

The effect of EPA/DHA on the expression of Adipoq and Lep and production of the adipokines was investigated in
WAT of mice with free access to chow (Table 2). At the whole-tissue level, no significant effect of the diet on expression of either Adipoq or Lep was detected. However, in adipocytes isolated from both depots, transcript levels for both adipokines were much higher than in the whole tissues, and Adipoq expression was stimulated by EPA/DHA, while Lep expression was unaffected. The stimulation of Adipoq expression was more pronounced in epididymal than in dorsolumbar fat (2.5- and 1.9-fold stimulation, respectively). Production of adiponectin was significantly increased by EPA/DHA in explants of epididymal but not dorsolumbar fat, while production of leptin was unchanged in the depots studied.

\section{Discussion}

This study demonstrates that caloric restriction and the intake of $n$-3 PUFA of marine origin both lead to weight loss and a decrease in adipose tissue. The latter treatment, corresponding to about $5.3 \%$ of total energy intake derived from EPA and DHA, led to a significant reduction of weight gain, involving preferential reduction of epididymal fat in the abdomen [1]. Of note, however, is the differential effect of these two treatments on glucose and lipid metabolism and systemic adipokines. While changes in circulating glucose and leptin were almost entirely mediated by caloric restriction, NEFA, insulin and adiponectin were regulated predominantly by dietary EPA and DHA, and triacylglycerols levels were decreased by both treatments. Various effects of caloric restriction and EPA/DHA respectively reflect modulation of metabolism in several tissues and all the interactions are difficult to dissect. However, as demonstrated in this report, one of the main effects of EPA/DHA is the stimulation of Adipoq expression in mature adipocytes and the production of adiponectin, mainly in epididymal fat, leading to increased plasma adiponectin levels.

Plasma levels of adiponectin decrease in obese humans [6]. In our study, however, the induction of adiponectin by EPA/DHA could not result from reduction of adiposity, 
since the strong decrease of fat content due to caloric restriction did not influence adiponectin levels. In contrast to adiponectin, leptin plasma levels were decreased dramatically by caloric restriction, but neither these nor Lep expression were affected by EPA/DHA, supporting the idea that circulating leptin correlates with adiposity and glucose metabolism in adipocytes [7]. The induction of adiponectin but not of leptin by EPA/DHA indicates that the mechanisms controlling the expression of genes for these two adipokines are different. As with thiazolidinediones, EPA and DHA may upregulate Adipoq by acting as ligands of peroxisome proliferator-activated receptor- $\gamma$, the transcriptional regulator interacting directly with Adipoq promoter [8]. The stimulation of Adipoq may also depend on the activation of AMPK, since AMPK in adipocytes stimulates Adipoq [9] and EPA and DHA activate hepatic AMPK [10].

In accordance with other studies in rodents fed high-fat diets and in healthy humans $[1,3]$, our insulin data indicate that EPA and DHA exert a large improvement in insulin sensitivity/action. Indeed, EPA/DHA prevented a rise in plasma insulin due to the cHF diet, since insulin levels in mice fed a standard chow diet were much lower than in the cHF mice, i.e. $0.59 \pm 0.10 \mathrm{ng} / \mathrm{ml}[1]$ (compare with Table 1). Our results suggest that protection against insulin resistance induced by a high-fat diet is at least partially mediated by adiponectin, but not leptin. Since EPA and DHA in humans were able to prevent but not reverse insulin resistance [3], it should be established whether the failure of dietary EPA and DHA to reverse type 2 diabetes is due to an inability to sufficiently induce adiponectin. Interestingly, an association between circulating adiponectin and plasma $n-3$ PUFA, and DHA in particular, was recently found in healthy humans [11].

In conclusion, we show for the first time that EPA and DHA stimulate Adipoq expression and increase the levels of circulating adiponectin, an effect that is relatively independent of food intake and body fat mass. Our findings are relevant for prevention and treatment of obesityassociated pathologies.
Acknowledgements This work was supported by grants from the Academy of Sciences (KJB5011410), Czech Science Foundation (303/05/2580) and Ministry of Education, Youth and Sports (1M6837805002) of the Czech Republic, as well as from the Wellcome Trust (070821/Z/03/Z), and from the European Commission (LSHM-CT-2004-005272), Research Project AVOZ50110509, and by Pronova Biocare (Norway).

\section{References}

1. Ruzickova J, Rossmeisl M, Prazak T et al (2004) Omega-3 PUFA of marine origin limit diet-induced obesity in mice by reducing cellularity of adipose tissue. Lipids 39:1177-1185

2. Ruxton CH, Reed SC, Simpson MJ, Millington KJ (2004) The health benefits of omega- 3 polyunsaturated fatty acids: a review of the evidence. J Hum Nutr Diet 17:449-459

3. Delarue J, LeFoll C, Corporeau C, Lucas D (2004) N-3 long chain polyunsaturated fatty acids: a nutritional tool to prevent insulin resistance associated to type 2 diabetes and obesity? Reprod Nutr Dev 44:289-299

4. Flachs P, Horakova O, Brauner P et al (2005) Polyunsaturated fatty acids of marine origin upregulate mitochondrial biogenesis and induce beta-oxidation in white fat. Diabetologia 48:2365-2375

5. Yamauchi T, Kamon J, Minokoshi Y et al (2002) Adiponectin stimulates glucose utilization and fatty-acid oxidation by activating AMP-activated protein kinase. Nat Med 8:12881295

6. Arita Y, Kihara S, Ouchi N et al (1999) Paradoxical decrease of an adipose-specific protein, adiponectin, in obesity. Biochem Biophys Res Commun 257:79-83

7. Wellhoener P, Fruehwald-Schultes B, Kern W et al (2000) Glucose metabolism rather than insulin is a main determinant of leptin secretion in humans. J Clin Endocrinol Metab 85:12671271

8. Iwaki M, Matsuda M, Maeda N et al (2003). Induction of adiponectin, a fat-derived antidiabetic and antiatherogenic factor, by nuclear receptors. Diabetes 52:1655-1663

9. Lihn AS, Jessen N, Pedersen SB, Lund S, Richelsen B (2004) AICAR stimulates adiponectin and inhibits cytokines in adipose tissue. Biochem Biophys Res Commun 316:853-858

10. Suchankova G, Tekle M, Saha AK, Ruderman NB, Clarke SD, Gettys TW (2005) Dietary polyunsaturated fatty acids enhance hepatic AMP-activated protein kinase activity in rats. Biochem Biophys Res Commun 326:851-858

11. Fernandez-Real JM, Vendrell J, Ricart W (2005) Circulating adiponectin and plasma fatty acid profile. Clin Chem 51:603609 